\title{
The MAP Mission
}

\author{
Edward L. Wright \\ Institute for Advanced Study, Princeton NJ 08540 and UCLA \\ Astronomy, PO Box 951562, Los Angeles CA 90095-1562, USA
}

\begin{abstract}
The Microwave Anisotropy Probe (MAP) is scheduled for launch in 2001. This mission will measure the anisotropy of the cosmic microwave background in five frequency bands from 22 to $90 \mathrm{GHz}$. The angular resolution varies with frequency from $0.93^{\circ}$ to $<0.23^{\circ}$. The sensitivity should reach a standard deviation of $20 \mu \mathrm{K}$ in each $0.3^{\circ}$ pixel on the sky. MAP will observe from the Earth-Sun $L_{2}$ point using a four-fold modulation scheme to minimize systematic errors. The 2-year planned duration will allow for 4 complete coverages of the sky. By observing the CMB angular power spectrum, MAP will tell us the initial conditions for the galaxy formation that produced the extragalactic infrared background.
\end{abstract}

\section{Introduction}

Observations of the anisotropy of the CMB (Cosmic Microwave Background) provide some of the earliest views into the history of our Universe. The photons of the CMB have traveled freely since the time of last scattering, about $t_{L S}=10^{5.5}$ years after the Big Bang. Prior to last scattering, photons were trapped in place by repeated scattering off electrons in the primordial plasma. Thus any inhomogeneity that existed prior to last scattering will have been converted into anisotropy and is thus observable. On angular scales larger than $2^{\circ}$, the structures we see on the surface of last scattering are larger than $c t_{L S}$, and are thus either primordial or created during an inflationary epoch. The detection by the COBE DMR of an anisotropy (Smoot et al. 1992, Wright et al. 1992, Bennett et al. 1992 and Kogut et al. 1992) on these large scales, that was consistent with a primordial density perturbation power spectrum $P(k)$ that was both scale-free (a power law form: $P(k) \propto k^{n}$ ) and scale-invariant (the Harrison-Zeldovich $n=1$ predicted by inflation), provided dramatic observational evidence in favor of inflationary dark-matter-dominated versions of the Big Bang model.

Physical processes acting prior to recombination will modify the CMB anisotropy on angular scales smaller than the angle subtended by the distance sound can travel between $t \approx 0$ and $t_{L S}$. The gravitational potential perturbations produced by dark matter density perturbations drive acoustic oscillations in the photon-baryon fluid. Recombination terminates the baryon-photon oscillations, and the relative phase between the baryon-photon density perturbations and the gravitational potential perturbations depends on the wavelength 


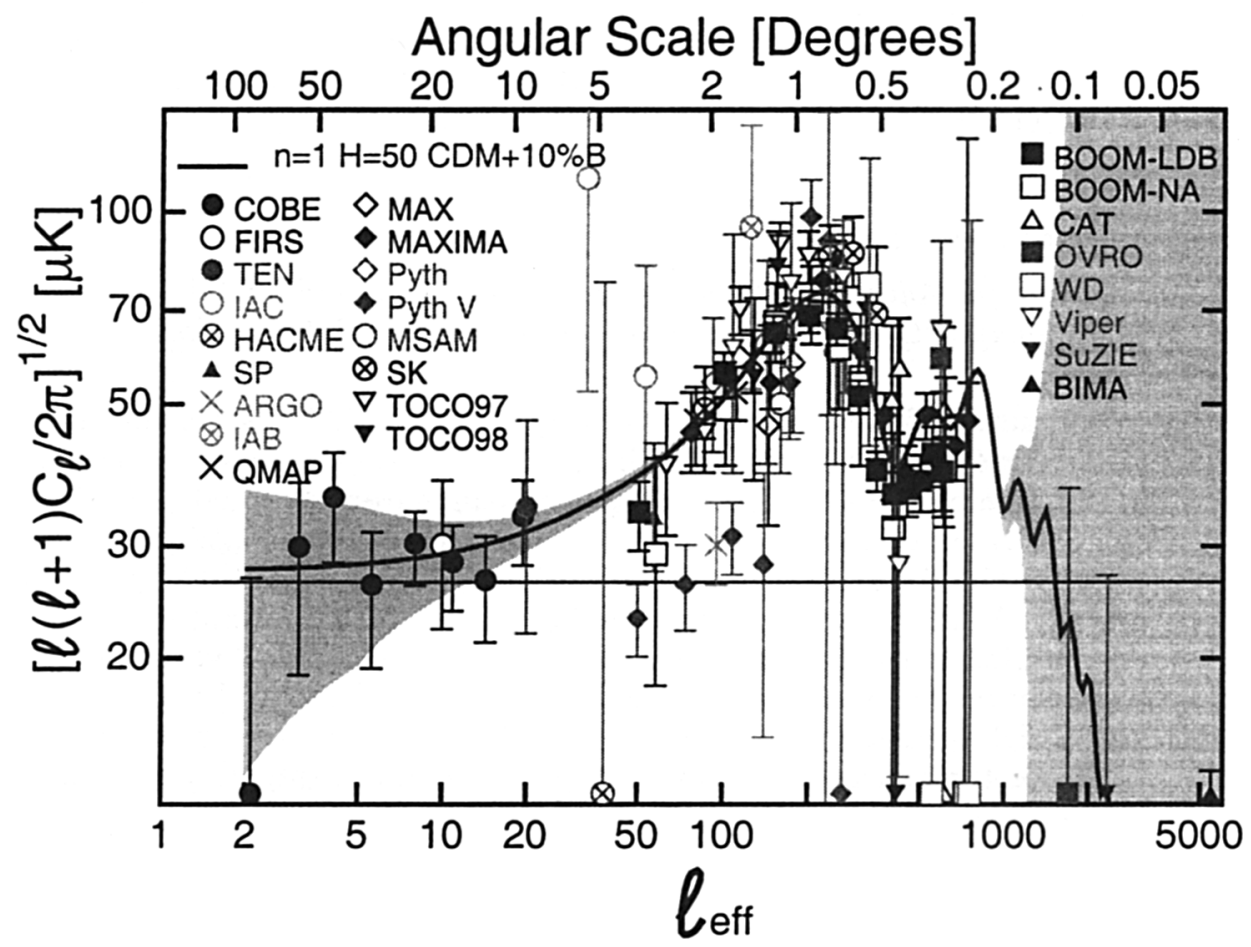

Figure 1. Data from 25 CMB anisotropy experiments. The $y$-axis gives the RMS temperature fluctuations in a bandwidth $\Delta \ell=\ell$ as a function of the spherical harmonic index $\ell$. The model curve is a standard CMB model with a slightly high baryon density.

of the perturbation, resulting in interference that leads to a series of peaks in the angular power spectrum (Bond \& Efstathiou 1987). Following the COBE DMR detection, a large number of experiments reported results at smaller angular scales, leading to a clear detection of the first of these acoustic peaks and approximate measurements of its position and amplitude. The latest of these results, from BOOMERanG and MAXIMA, have covered $3 \%$ of the sky with angular resolutions of $0.2^{\circ}$, which are much better than the $7^{\circ}$ resolution of the COBE DMR. Figures 1 and 2 show the current state of knowledge from these experiments.

In addition to these ground-based and balloon-borne experiments, two new satellites are being built to study the CMB. In the US, the MAP ${ }^{1}$ project is in the final stages of integration and test, aiming for a launch currently scheduled for spring 2001. In Europe, the much more ambitious Planck ${ }^{2}$ project will be launched with the FIRST far-infrared telescope no sooner than 2007.

\footnotetext{
${ }^{1}$ http://map.gsfc.nasa.gov

${ }^{2}$ http://astro.estec.esa.nl/SA-general/Projects/Planck/
} 


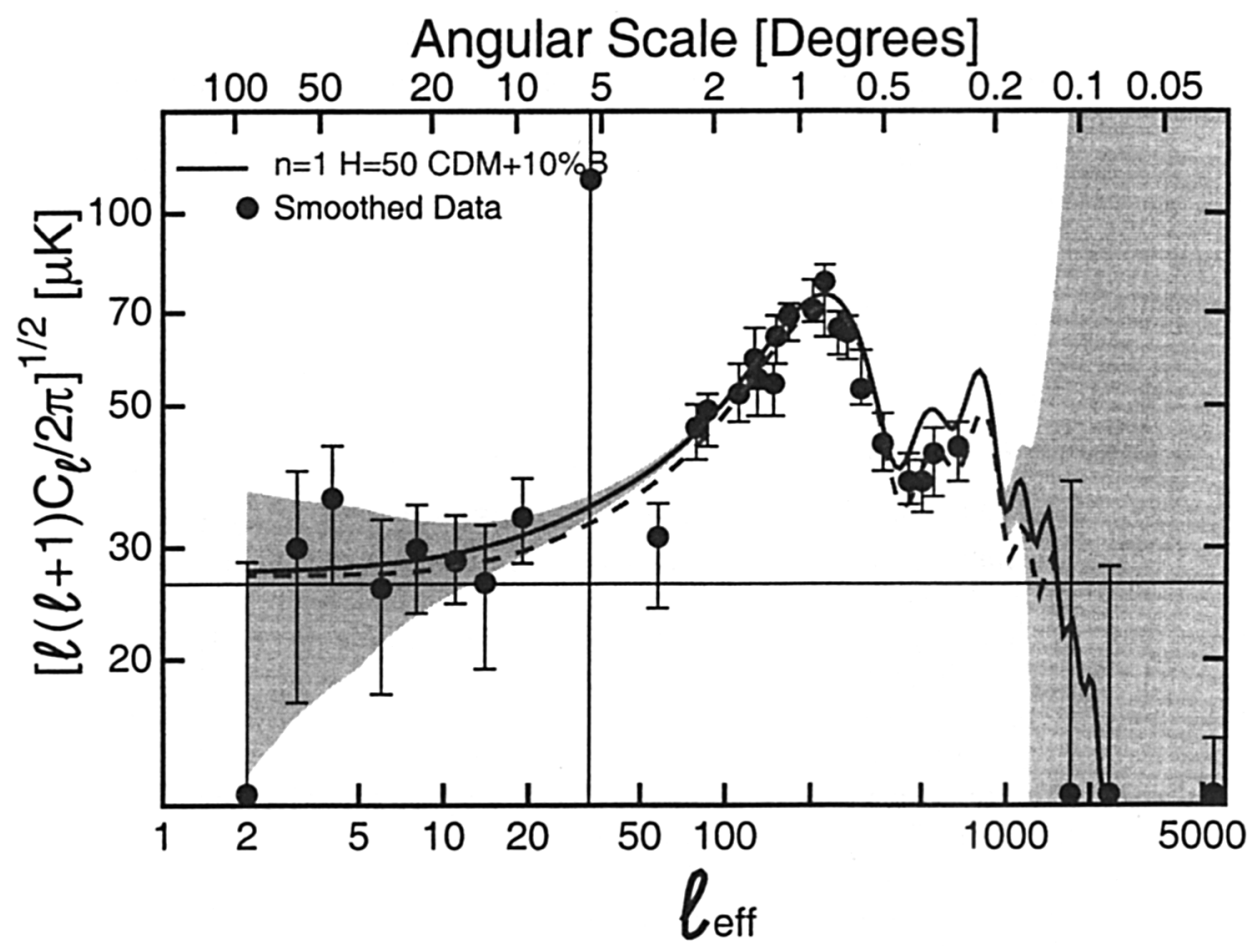

Figure 2. The data from Figure 1 smoothed with a $25 \%$ wide running median filter. A new dashed curve showing a model with $H_{\circ}=52$, baryon density $\Omega_{B} h^{2}=0.03$, CDM density $\Omega_{C D M} h^{2}=0.19$ and cosmological constant $\Omega_{\Lambda}=0.19$ has been added.

\section{MAP Mission Description}

During the proposal process, the MAP Principal Investigator, Charles L. Bennett, and the members of the science team recognized the need to scan the modulated sky signals at frequencies above the $1 / f$-noise knee of the detectors. Although bolometers were considered, HEMT (high electron mobility transistor) radiometers were chosen for simplicity, in the spirit of better, faster, cheaper. HEMT amplifiers work directly at the $20-100 \mathrm{GHz}$ frequencies of interest to MAP with very low noise and high bandwidths, but have a fairly high $1 / f$ knee when used as total power radiometers. Therefore, experiments that use total power HEMT radiometers must scan the sky at high angular rates. Thus, the QMAP experiment (de Oliveira-Costa et al. 1998) used a scan mirror to sweep its beams across the sky at $200^{\circ} / \mathrm{sec}$.

MAP was required to scan through each pixel in many different directions. Scanning in only one direction leads to stripes. When combined with $1 / f$ noise, scanning in only one direction leads to terrible stripes (Wright 1996). Thus MAP needed to scan in a complicated cycloidal pattern like the COBE scan pattern. This implied that a simple spinning spacecraft would not be satisfactory, and that a continuously slewing 3 -axis stabilized spacecraft was needed. But a 3-axis 
stabilized spacecraft that could slew continuously at $200 \% \mathrm{sec}$ was not possible within the MIDEX budget constraints, so MAP became a two-sided chopping instrument.

After these decisions, MAP has the same complement of 4 different modulations that COBE used:

- A fast chop between two beams

- A rotation around a spin axis

- A precession of the spin axis around the anti-solar point

- A rotation of the whole scan pattern around the sky once per year

Furthermore, MAP chose an orbit at the Earth-Sun $L_{2}$ Lagrange point, along the Earth-Sun line but $1.5 \times 10^{6} \mathrm{~km}$ further from the Sun than the Earth. This orbit eliminates the two largest contributors to systematic errors in the COBE data: the Earth's magnetic field and the thermal emission from the Earth's limb.

The MAP radiometer front ends are passively cooled to $\sim 95 \mathrm{~K}$. MAP uses pseudo-correlation differential radiometers in which all the amplification prior to the square law detectors is at the observation frequency. There are no local oscillators or intermediate frequency (IF) amplifiers. MAP will map the entire sky in 5 bands centered at $22,30,40,60 \& 90 \mathrm{GHz}$. The beam sizes are $0.93^{\circ}, 0.68^{\circ}, 0.47^{\circ}, 0.35^{\circ}$ and $<0.23^{\circ}$ respectively, after allowing for the beamsmearing caused by scanning during sampling. The sensitivity will give a noise level of $\sim 35 \mu \mathrm{K}$ after two years in each of 393,216 pixels at each frequency. The actual number of pixels in the MAP maps will be larger and the noise per pixel correspondingly higher. Both total intensity and linear polarization will be measured in all channels.

\section{Status}

The MAP spacecraft and instrument have been integrated into the MAP observatory, and the whole satellite will undergo EMI, EMC, acoustic, vibration, thermal vacuum, functional and thermal balance testing in the fall of 2000 . If all goes well, MAP will be ready for launch in spring 2001. After launch it will take MAP about 3 months to reach its station at $L_{2}$, and 6 more months to map the sky for the first time. After 9 months of data analysis, the first results from MAP covering $100 \%$ of the sky should be released 18 months after launch.

\section{Prospects}

The light gray bands on Figures 1 and 2 show the expected noise performance of MAP after two years of data gathering. MAP should provide a cosmic variance limited measurement of the angular power spectrum of the CMB anisotropy through the first two acoustic peaks, and greatly improve our knowledge of the state of the Universe 300,000 years after the Big Bang. MAP should also detect the temperature-polarization cross-correlation. Thus, MAP will tell us the initial conditions for the galaxy formation process that produced the Cosmic Infrared Background between $t_{L S}$ and now. 
Acknowledgments. I would like to thank the other members of the MAP science team: C. Bennett, M. Halpern, G. Hinshaw, N. Jarosik, A. Kogut, M. Limon, S. Meyer, L. Page, D. Spergel, G. Tucker, D. Wilkinson, \& E. Wollack. This research has been supported by NASA, and at the IAS by the National Science Foundation grant number PHY-0070928 and the Ambrose Monell Foundation.

\section{References}

Bennett, C. L., et al. 1992, ApJ, 396, L7

Bond, J. R., \& Efstathiou, G. 1987, MNRAS, 226, 655

Kogut, A., et al. 1992, ApJ, 401, 1

de Oliveira-Costa, A., Devlin, M., Herbig, T., Miller, A., et al. 1998, ApJ, 509, L77

Smoot, G. F., et al. 1992, ApJ, 396, L1

Wright, E. L., et al. 1992, ApJ, 396, L13

Wright, E. L. 1996, http://xxx.lanl.gov/abs/astro-ph/9612006

\section{Discussion} ters?

Michael Hauser: How well will MAP determine the cosmological parame-

Ned Wright: That depends on the parameter and the priors. The position of the first peak, which determines $\Omega_{\text {total }}$, will be measured to $<1 \% . \Lambda$ and $H_{0}$ should be determined to $\sim 10 \%$, which would provide an independent test of the "accelerating universe" implied by supernova data.

Charley Lineweaver: Two questions: 1 . Your estimate of cosmic variance at low $\ell$ is larger than other estimates I have seen. Is someone making a mistake? 2 . What is your calibration accuracy?

Wright: 1 . My chart shows bins at $\Delta \ell / \ell=20 \%$ on a log - log plot showing $C_{\ell}$, not $\Delta T$. This may have led to an apparently large cosmic variance. 2 . Calibration will be done relative to the dipole to $<1 \%$ accuracy. COBE made an absolute physical determination of the dipole to $<1 \%$. 